(C) 2006 IEEE. Reprinted, with permission, from Youguang Guo, Robust Optimization of Multilayer Conductors of HTS AC Cable Using PSO and Perturbation Analysis . Industry Applications Conference, 2006. 41st IAS Annual Meeting. Conference Record of the 2006 IEEE (Volume:1 ), October 2006. This material is posted here with permission of the IEEE. Such permission of the IEEE does not in any way imply IEEE endorsement of any of the University of Technology, Sydney's products or services. Internal or personal use of this material is permitted.

However, permission to reprint/republish this material for advertising or promotional purposes or for creating new collective works for resale or redistribution must be obtained from the IEEE by writing to pubs-permissions@ieee.org. By choosing to view this document, you agree to all provisions of the copyright laws protecting it 


\section{Robust Optimization of Multilayer Conductors of HTS AC Cable Using PSO and Perturbation Analysis}

\author{
Shuhong Wang, Jie Qiu, Zhen Zhao and Xinying Liu \\ Faculty of Electrical Engineering \\ Xi'an Jiaotong University \\ Xi'an, 710049 China \\ shwang@mail.xjtu.edu.cn
}

\author{
Jian Guo Zhu, Youguang Guo and Zhi Wei Lin \\ Faculty of Engineering \\ University of Technology, Sydney \\ Sydney, P.O. Box 123, NSW 2007, Australia \\ Joe@eng.uts.edu.au
}

\begin{abstract}
For a High Temperature Superconducting (HTS) cable, a non-uniform AC current distribution among the multilayer conductors gives rise to increased AC losses. To get a uniform current distribution among the multilayer conductors, a constrained optimization model is constructed with continuous and discrete variables, such as the winding angle, radius and the winding direction of each layer. Under the constraints of the mechanical properties and critical current of the tape, the Particle Swarm Optimization (PSO) algorithm is employed for structural parameter optimization in both warm and cold dielectric type HTS cables. A uniform current distribution among layers is realized by optimizing the structural parameters. The perturbation analysis is employed to evaluate the parameters after optimization. It is found that the robust stabilizations are different among the various optimal results. The PSO is proved to be a more powerful tool than the Genetic Algorithm (GA) for structural parameter optimization.
\end{abstract}

Keywords-Current distribution, high temperature superconducting (HTS) cable, particle swarm optimization (PSO), perturbation analysis.

\section{INTRODUCTION}

A high Temperature Superconducting (HTS) cable is an important part of HTS power applications. For HTS cables different design concepts of cold and warm temperature dielectrics have been proposed [1]. In a cable with warm dielectric, the design is relatively simple. The main advantage of the warm dielectric type HTS cable is that its impedance matches that of a conventional cable, whose diameter is similar to the warm dielectric type HTS cable, and it will not affect the flow of power though other cables. At its core is a duct through which the liquid nitrogen flows. The duct is surrounded by HTS wire, which is insulated with a dielectric sleeve. Over the dielectric is a protective sheath. In a cable with cold dielectric, the core consists of a flexible stainless steel (STS) former, a multilayer HTS conductor, an electrical insulation, a multilayer HTS shield, and a mechanical protection layer [2]. The cold dielectric type configuration has several advantageous features compared to the warm dielectric type configuration, such as smaller diameter, reduction of leakage of the magnetic field and reduction of the inductive component in impedance using a superconducting shielding layer [1]

For the large current transmission, a HTS cable has a multilayer structure which consists of parallel connected tapes, twisted in each layer. Due to different inductance among layers, the currents flowing in the layers are not the same, as demonstrated by a number of experiments performed on the cable conductors [3], [4]. Hence, the control of the current distribution among layers is an important issue for design and optimization of a HTS cable conductor because it is related to current transmission capacity and power losses.

Usually, for an AC HTS cable, the inductive impedance is much greater than the joint resistance, and thus, the current distribution among layers is substantially determined by the inductive impedances of layers. The distribution of inductive impedances is however dependent on the structural parameters of the cable conductor, such as the radius, winding pitch and winding direction of a layer. The main method to obtain a uniform current distribution is to alternate the inductive impedances of layers by adjusting the structural parameters of the cable conductors. The validity of this method has been verified by the experimental results of current distribution [4] [5].

Practical performances of HTS cable may contain a degree of parameter perturbations, possibly caused by manufacturing error or the properties of superconducting tape, such as shrinkage at low-temperature. These perturbed parameters may distort the performances of HTS cable [6].

This paper presents a method to achieve a uniform current distribution in both warm and cold dielectric type HTS cables by adjusting the structural parameters of the conductor layers using the Particle Swarm Optimization (PSO) algorithm. The influences of parameter perturbations on the optimized multilayer conductor structures are also analyzed. A comparison between PSO and Genetic Algorithm (GA) is conducted.

\section{THE MODEL OF HTS CABLE}

\section{A. Warm Dielectric Type HTS Cable}

A 4-layer HTS cable is simulated. The schematic view of the simulated cable is given in Fig.1.

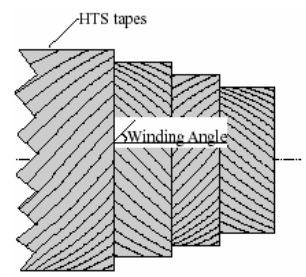

Figure 1. Schematic view of a 4-layer warm dielectric type HTS cable. 
The modeling of a warm dielectric type HTS cable by an equivalent circuit as shown in Fig.2, which takes into account the resistive behaviors of the superconductor, has been presented in an earlier paper [4].

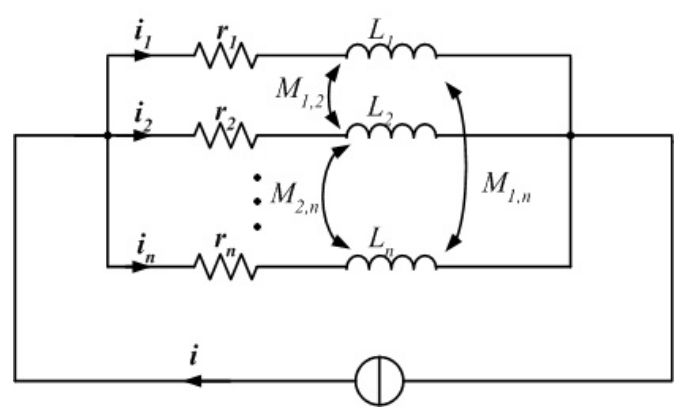

Figure 2. Equivalent circuit model of a warm dielectric type HTS cable.

Here $r_{i}$ and $L_{i}$ are the resistance and self-inductance of the $i$-th layer, respectively, and $M_{i j}$ is the mutual inductance between the $i$-th and $j$-th layers. According to [3], the self and mutual inductances per unit length can be given by

$$
L_{i}=\frac{\mu_{0}}{4 \pi}\left(\tan ^{2} \beta_{i}+2 \ln \frac{D}{R_{i}}\right) \quad i=1,2, \cdots, n
$$

and

$$
M_{i j}=\frac{\mu_{0}}{2 \pi}\left(\frac{a_{i} a_{j}}{2} \frac{R_{i}}{R_{j}} \tan \left(\beta_{i}\right) \tan \left(\beta_{j}\right)+\ln \frac{D}{R_{j}}\right) \quad i \neq j
$$

where $R_{i}$ and $R_{j}\left(R_{i}<R_{j}\right)$ are the radii, $\beta_{i}$ and $\beta_{j}$ the winding angles, $\alpha_{i}$ and $\alpha_{j}$ the constants $(-1$ or +1$)$ taking into account the relative winding directions of the $i$-th and $j$-th layers, respectively, and $D$ is the distance between the cable and the return lead.

The resistance per unit length is expressed as a power law using the global parameters:

$$
r_{e, i}\left(I_{i}\right)=\text { const } \times \frac{\left|I_{i}\right|^{n-1}}{I_{c, i}^{n}}
$$

where const $=10^{-4} \mathrm{~V} / \mathrm{m}$, and $I_{c i}$ is the critical current of the $i$-th layer.

\section{B. Cold Dielectric Type HTS Cable}

Fig.3 shows the structure of the single-phase cold dielectric type HTS power cable, which consists of four layers of conductor and two layers of shield used Ag/Bi-2223 tapes [7].

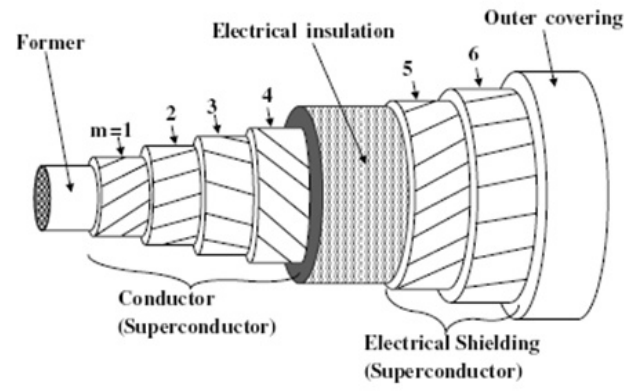

Figure 3. Schematic diagram of the cold dielectric type HTS cable.
The equivalent circuit of the cold dielectric type HTS cable consisting of $m$ layers of phase conductor and $n-m$ layers of shield is shown in Fig.4.

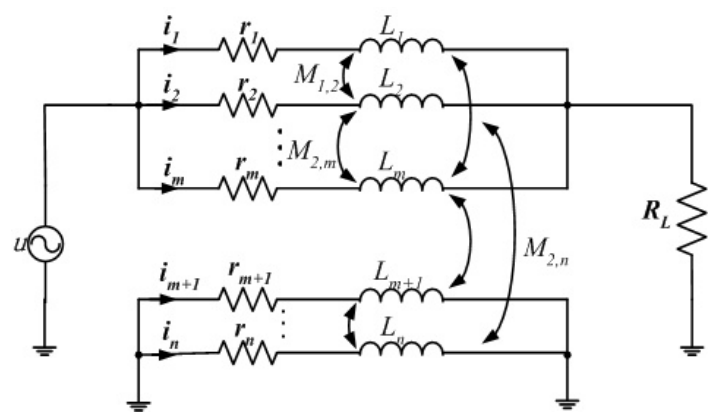

Figure 4. Equivalent circuit model of a cold dielectric type HTS cable conductor.

Here the resistance $r_{i}$, self-inductance $L_{i}$ and the mutual inductance $M_{i j}$ can be described in (1), (2) and (3) respectively.

\section{THE MODEL OF OPTIMIZATION}

\section{A. Objective Function}

According to (1) and (2), the self- and mutual- inductances of a conductor layer are correlated with the winding angle $\beta$, the winding direction $\alpha$ and the radius $R$ of the layer. So the parameters mentioned above are chosen as the optimized variables. If there are $n$ layers in the cable, the optimized variables can be expressed as a vector:

$$
\boldsymbol{X}=\left[\beta_{1}, a_{1}, R_{1}, \beta_{2}, a_{2}, R_{2}, \cdots, \beta_{n}, a_{n}, R_{n}\right]
$$

Without quenching, the objective function of optimization for the warm dielectric type HTS cable is derived to find a solution for a uniform current distribution in a HTS cable conductor layer.

$$
\min f(\boldsymbol{X})=\sum_{i=1}^{n-1} \sum_{j=i+1}^{n}\left|I_{i x}(\boldsymbol{X})-I_{j x}(\boldsymbol{X})\right|+\sum_{i=1}^{n-1} \sum_{j=i+1}^{n}\left|I_{i y}(\boldsymbol{X})-I_{j y}(\boldsymbol{X})\right|
$$

where $I_{i x}(\boldsymbol{X})$ and $\mathrm{I}_{i y}(\boldsymbol{X})$ are the real and imaginary components of current $\dot{I}_{i}(\boldsymbol{X})$ in the $i$-th layer. $\dot{I}_{i}(\boldsymbol{X})$ as a function of the parameter vector $\boldsymbol{X}$ can be derived from (3). The current distribution among layers should become more uniform when $f(X)$ is closer to a minimal value.

For the cold dielectric type HTS cable, the purpose of optimization is to achieve the current of the phase conductors and that of the shields uniform respectively. The objective function of optimization is given by

$$
\begin{array}{r}
\min f(\boldsymbol{X})=\sum_{i=1}^{m-1} \sum_{j=i+1}^{m}\left|I_{i x}(\boldsymbol{X})-I_{j x}(\boldsymbol{X})\right|+\sum_{i=1}^{m-1} \sum_{j=i+1}^{m}\left|I_{i y}(\boldsymbol{X})-I_{j y}(\boldsymbol{X})\right| \\
+\sum_{i=m+1}^{n-1} \sum_{j=i+1}^{n}\left|I_{i x}(\boldsymbol{X})-I_{j x}(\boldsymbol{X})\right|+\sum_{i=m+1}^{n-1} \sum_{j=i+1}^{n}\left|I_{i y}(\boldsymbol{X})-I_{j y}(\boldsymbol{X})\right|
\end{array}
$$




\section{B. Constraints}

1) Constraints of the mechanical properties

Firstly, considering the mechanical properties of a tape, such as the tensile strain characteristic and the bending strain characteristic, the optimum program should satisfy the following two conditions: (1) the tensile strain of tape must be less than the critical tensile strain, and (2) the bending strain must be less than the critical bending strain [8]. Hence, the constraints of mechanical properties can be expressed as

$$
\left\{\begin{array}{l}
\beta_{i}-\arcsin \left(\frac{2 R_{i} \varepsilon_{c b}}{t}\right)^{1 / 2} \leq 0 \\
\beta_{i}-\arcsin \left(\frac{\varepsilon_{c t}+\varepsilon_{p}-\varepsilon_{f c}}{\varepsilon_{p}-\varepsilon_{r}}\right)^{1 / 2} \geq 0
\end{array} \quad i=1,2, \cdots, n\right.
$$

where $\varepsilon_{c b}$ and $\varepsilon_{c t}$ are the critical bending and tensile strain of the tape at $77 \mathrm{~K}, \varepsilon_{p}$ and $\varepsilon_{f c}$ are the thermal shrinkages of the winding pitch and the tape, respectively, $\varepsilon_{r}$ is the radial thermal shrinkage of the former, and $t$ the thickness of the tape.

\section{2) Constraints of radii}

The constraints of radii can be expressed as

$$
\left\{\begin{array}{l}
R_{1}-\frac{D_{\min }}{2}+\left(t_{f}+\frac{t}{2}\right) \geq 0 \\
R_{i+1}-R_{i}-\left(t_{f}+t\right) \geq 0 \quad i=1,2, \cdots, n-1 \\
\frac{D_{\max }}{2}-R_{i}-(n-i)\left(t_{f}+t\right)-\frac{t}{2} \geq 0 \quad i=1,2, \cdots n
\end{array}\right.
$$

where $D_{\min }$ and $D_{\max }$ are used to limit the inner and outer diameters of the cable conductors, and $t_{f}$ is the thickness of the dielectric between layers.

\section{3) Constraints of critical current}

These constraints are used to restrict the currents in layers below their critical currents, and can be expressed as

$$
I_{i}<N_{i} I_{c} k_{1} k_{2} k_{3} k_{4} \quad i=1,2, \cdots, n
$$

where $N_{i}$ is the number of tapes wound on the i-th layer, $I_{c}$ the mean of critical currents of HTS tapes in the cable, $k_{1}, k_{2}$ and $k_{3}$ are the deteriorations of the critical current considering the magnetic field and the temperature, manufacture, and the thermal cycles, respectively, and $k_{4}$ is the design safety margin.

\section{The Particle SWARM Optimization MethoD}

Particle swarm optimization (PSO) is a population based stochastic optimization technique developed by Kennedy and Eberhart in 1995 and inspired by the social behavior of birds flocking and fish schooling [9]. In PSO, each potential solution called a 'particle', flies in the problem search hyperspace looking for the optimal position. As time passes, a particle adjusts its position according to its own 'experience', as well as according to the experience of neighboring particles.

Suppose that the search space is D-dimensional, and each particle is treated as a point in a D-dimensional space. The position of the i-th particle of the swarm can then be expressed as a vector $X_{i}(t)=\left(X_{i, 1}(t), X_{i, 2}(t), \ldots, X_{i, D}(t)\right)$. The velocity (position change) of this particle can be represented by another vector $V_{i}(t)=\left(V_{i, 1}(t), V_{i, 2}(t), \ldots, V_{i, D}(t)\right)$. The i-th particle also maintains a memory of its previous best position in vector pbest $_{i}=\left(\right.$ pbest $_{i, 1}$, pbest $_{i, 2}, \ldots$, pbest $\left._{i, D}\right)$. In each iterative step, the gbest is designated as the index of the best particle in the swarm. Subsequently, the swarm is manipulated according to the following two equations [10]:

$$
\begin{gathered}
V_{i, d}(t)=w V_{i, d}(t-1)+c_{1} r_{1} \times\left(\text { pbest }_{i, d}-X_{i, d}(t-1)\right) / \Delta t \\
+c_{2} r_{2} \times\left(\text { gbest }_{d}-X_{i, d}(t-1)\right) / \Delta t \\
X_{i, d}(t)=X_{i, d}(t-1)+V_{i, d}(t) \times \Delta t
\end{gathered}
$$

where $d=1,2, \ldots, D, i=1,2, \ldots, N, N$ is the size of the swarm, $c_{1}$ and $c_{2}$ are two positive constants, namely social and cognitive parameters, $r_{1}$ and $r_{2}$ two random numbers distributed within the range $[0,1], t$ is the iteration number, $\Delta t=1$, and $w$ is inertia weight.

Equation (9) is used to update the particle's new velocity according to its previous velocity and the distances of its current position from both its own best historical position and its neighbors' best position in every iterative step. Then the particle flies towards a new position according to (10).

\section{STRUCTURAL PARAMETERS OPTIMIZATION USING PSO}

The structural optimum design of a HTS cable is that a series of advanced parameters are obtained within the kinds of constraints, and thus the current distribution becomes as uniform as possible with these parameters. In this paper, both a 4-layer warm dielectric type HTS cable and a cold dielectric type HTS cable composed of 4-layer conductor and 2-layer shield are optimized by PSO respectively. The parameters are given as: $c_{1}=2.8$ and $c_{2}=1.3$. The adaptive inertia weight $w$ is defined as

$$
w=\left(w_{\text {start }}-w_{\text {end }}\right) C^{\text {gen }}+w_{\text {end }}
$$

where the constant $C=0.999$, gen is the current number of evolution iteration, the start value of inertia $w_{\text {start }}=1.43$, and the end value of inertia $w_{\text {end }}=0.1$. The inertia weight decreases from $w_{\text {start }}$ to $w_{\text {end }}$. It can facilitate an initial global exploration and dynamically investigate the refined solution.

\section{A. Warm Dielectric Type HTS Cable}

In Table I, the structural parameters of the 4-layer warm dielectric type HTS cable are shown in two cases: before and after optimization.

TABLE I. StRUCTURAL PARAMETERS OF WARM Dielectric TyPE HTS CABLE

\begin{tabular}{|c|c|c|c|c|c|c|}
\hline $\begin{array}{c}\text { Layer } \\
\text { Index }\end{array}$ & $a_{i}^{\Delta}$ & $\beta_{i}^{\Delta}$ & $R_{i}^{\Delta}(\mathbf{m m})$ & $a_{i}^{*}$ & $\beta_{i}^{*}$ & $R_{i}^{*}(\mathbf{m m})$ \\
\hline 1 & +1 & $27.0^{\circ}$ & 20.50 & +1 & $43.2^{\circ}$ & 20.00 \\
\hline 2 & -1 & $27.0^{\circ}$ & 21.00 & +1 & $24.6^{\circ}$ & 20.35 \\
\hline 3 & +1 & $27.0^{\circ}$ & 21.50 & -1 & $26.8^{\circ}$ & 21.34 \\
\hline 4 & -1 & $27.0^{\circ}$ & 22.00 & -1 & $43.0^{\circ}$ & 21.87 \\
\hline
\end{tabular}

Note: $\Delta$ represents before optimization,

* represents after optimization,

$\alpha$ is the winding direction,

$\beta$ the winding angle,

and $R$ the radius. 
Fig.5 and Fig. 6 are obtained based on the data in Table I. In these figures, it is shown that the currents before optimization differ greatly in both the amplitude and phase angle, but the optimized currents become uniform. Especially, as the peak of total cable current is pushed up to $1000 \mathrm{~A}$, the equivalent resistances of the layers increase as the outer layer current increases to the vicinity of the critical current, and the current waveforms before optimization are severely distorted. After optimization, the current waveforms become sinusoidal.

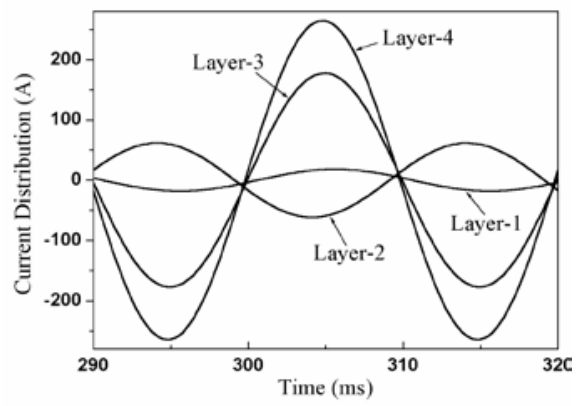

(a) Before optimization

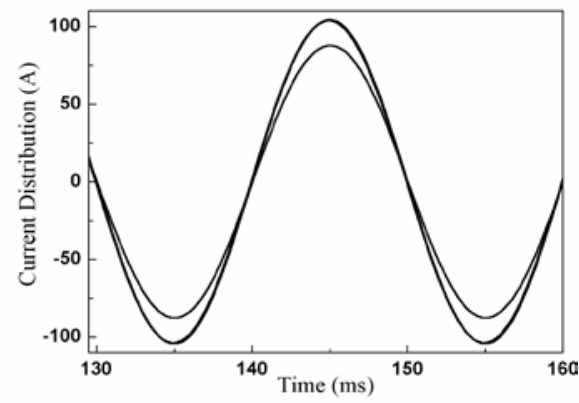

(b) After optimization

Figure 5. Current Distribution (the peak of total cable current is 400A).

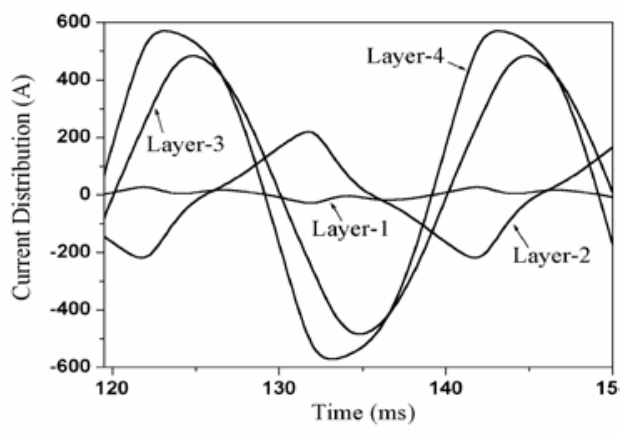

(a) Before optimization

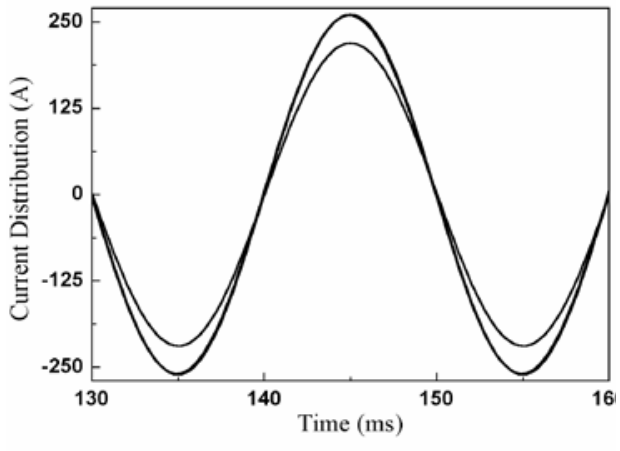

(b) After optimization

Figure 6. Current Distribution (the peak of total cable current is 1000A).

The AC losses are calculated by the method described in [11]. Fig.7 illustrates a comparison of the AC losses calculated before and after optimization. As shown, the optimization process can reduce significantly the AC losses of the cable.

Figure 7. Comparison of AC losses in warm dielectric type HTS cable before and after optimization.

\section{B. Cold Dielectric Type HTS Cable}

In Table $\Pi$, the structural parameters of the cold dielectric type HTS cable, which is composed of 4-layer conductor and 2-layer shield, are shown in two cases: before and after optimization.

TABLE II. Structural PARAMETERS OF THE COLD DiElEctric TyPE HTS CABLE

\begin{tabular}{|c|c|c|c|c|c|c|}
\hline $\begin{array}{c}\text { Layer } \\
\text { Index }\end{array}$ & $a_{i}^{\Delta}$ & $\beta_{i}^{\Delta}$ & $R_{i}^{\Delta}(\mathbf{m m})$ & $a_{i}{ }^{\Delta}$ & $\beta_{i}^{*}$ & $R_{i}^{*}(\mathbf{m m})$ \\
\hline 1 & +1 & $27.0^{\circ}$ & 10.0 & +1 & $25.8^{\circ}$ & 10.0 \\
\hline 2 & +1 & $27.0^{\circ}$ & 10.45 & +1 & $14.0^{\circ}$ & 10.5 \\
\hline 3 & -1 & $27.0^{\circ}$ & 10.90 & -1 & $8.5^{\circ}$ & 11.2 \\
\hline 4 & -1 & $27.0^{\circ}$ & 11.35 & -1 & $29.7^{\circ}$ & 11.9 \\
\hline 5 & +1 & 27.0 & 18.50 & +1 & 24.1 & 19.0 \\
\hline 6 & +1 & 27.0 & 18.95 & +1 & 8.5 & 20.8 \\
\hline
\end{tabular}

Note: The definition of $\Delta, *, \alpha, \beta$ or $R$ is similar to that in Table I. 
The length of the cable for calculation is chosen to be 100 $\mathrm{m}$, the AC voltage source $u$ is $10 \mathrm{kV}(\mathrm{rms})$, and the load $R_{L}$ is $10 \Omega$. The current distributions according to the parameters before and after optimization are shown in Fig.8 (a) and (b) respectively. In these figures, it is found that the optimized currents become uniform. The phase of shield current is opposite to that of the phase conductor current, and the magnitude of shield current is almost the same as that of the phase conductor current.

Fig.9 demonstrates that the AC losses of the cold dielectric type HTS cable are reduced significantly after optimization.

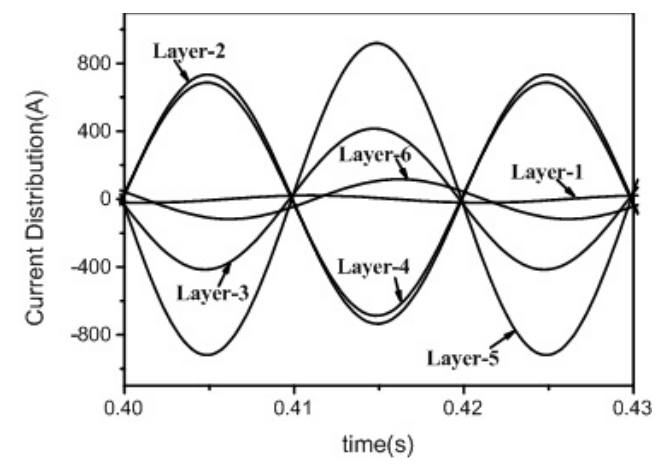

(a)

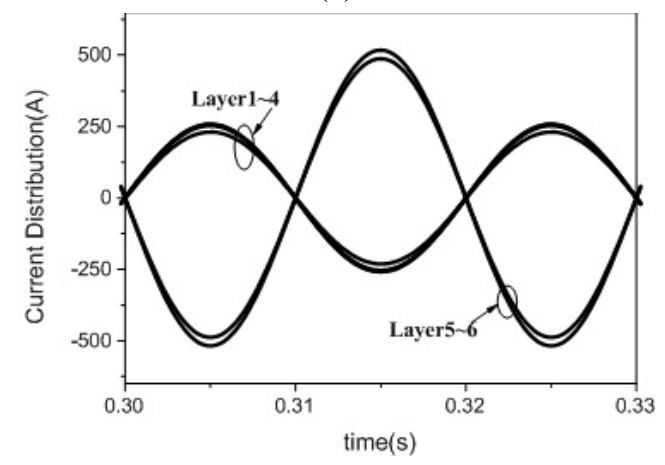

(b)

Figure 8. Current distribution in the cold dielectric type HTS cable.

\section{PERTURBATION ANALYSIS OF OptiMIZED PARAMETERS}

After optimization, the robust stabilization of structural parameters is important for the design of HTS cable. In this paper, the perturbation analysis is conducted to evaluate the influence of the distorted structure parameters. The current relative error of the $i$-th layer

$$
E_{c v, i}=\frac{\left|I_{\text {ave }}-I_{\text {err }, i}\right|}{I_{\text {ave }}} \times 100 \%
$$

is introduced to investigate the effect of perturbed parameters on the current distribution, where $I_{a v e}$ is the average value of the currents of the layers obtained through the optimized parameters, and $I_{e r r, i}$ the current of the $i$-th layer with the perturbed structure parameters.

\section{A. Warm Dielectric Type HTS Cable}

Three types of 4-layer warm dielectric type HTS cable with different optimal parameters are shown in Table III. The perturbations are performed on the winding angle and the radius in a certain range. The current relative error, $E_{c v}$, is calculated in the following two cases: (1) only perturbation of the winding angle, and (2) only perturbation of the radius.

TABLE III. THERE TYPES OF WARM DIELECTRIC TYPE HTS CABLE CONDUCTORS

\begin{tabular}{|c|c|c|c|c|c|}
\hline \multicolumn{2}{|c|}{ Layer Index } & NO.1 & NO.2 & NO.3 & NO.4 \\
\hline \multirow{3}{*}{ Cable 1 } & $\alpha$ & +1 & +1 & -1 & -1 \\
& $\beta$ & $43.2^{\circ}$ & $24.6^{\circ}$ & $26.8^{\circ}$ & $43.0^{\circ}$ \\
& $R / m m$ & 20.00 & 20.35 & 21.34 & 21.87 \\
\hline \multirow{3}{*}{ Cable 2 } & $\alpha$ & -1 & -1 & +1 & +1 \\
& $\beta$ & $39.7^{\circ}$ & $24.6^{\circ}$ & $26.2^{\circ}$ & $40.3^{\circ}$ \\
& $R / m m$ & 20.00 & 20.35 & 21.43 & 21.87 \\
\hline \multirow{3}{*}{ Cable 3 } & $\alpha$ & -1 & -1 & -1 & -1 \\
& $\beta$ & $27.5^{\circ}$ & $28.0^{\circ}$ & $31.7^{\circ}$ & $34.1^{\circ}$ \\
& $R / m m$ & 20.00 & 20.35 & 21.42 & 21.86 \\
\hline
\end{tabular}

The maximum value of $E_{\mathrm{cv}}$ in all cases is defined as $E_{\max }$. For Case 1, the relationship of $E_{\max }$ and winding angle perturbation is depicted in Fig.10 (a). In Fig.10 (b), $E_{\max }$ is shown as a function of radius perturbation in Case 2.

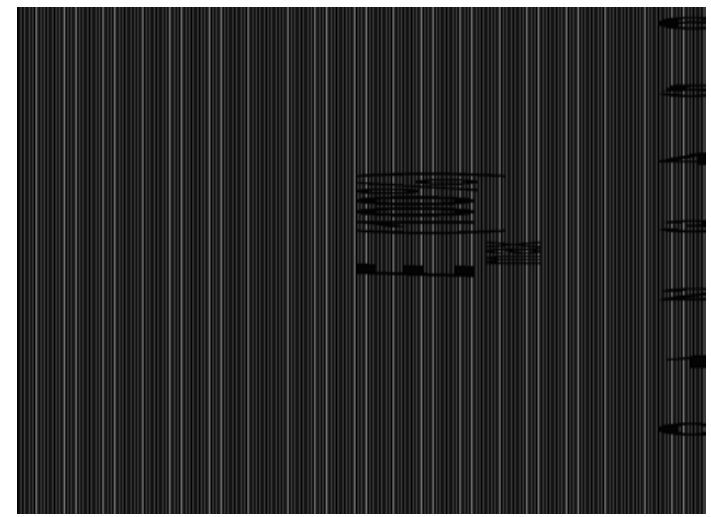

(a) Perturbation of the winding angle
Figure 9. Comparison of AC losses in cold dielectric type HTS cable before and after optimization 


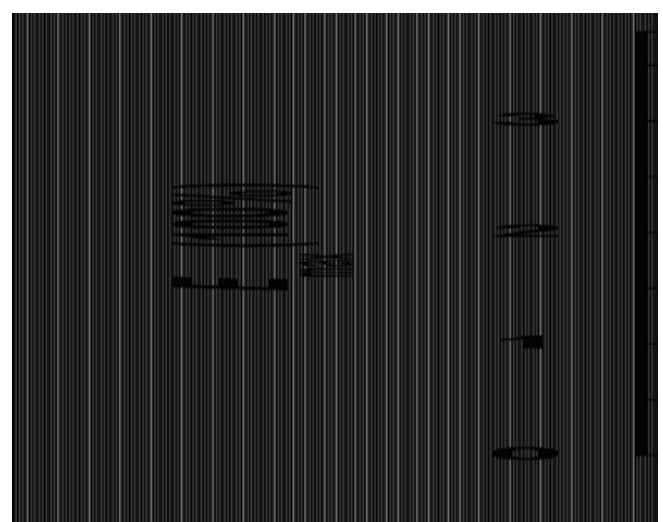

(b) Perturbation of the radius

Figure 10. Influence of parameter perturbation on current distribution in the warm dielectric type HTS cable.

The curves in Fig.10 reveal that $E_{\max }$ of Cable 1 is evidently less than that of Cables 2 and 3 . It can be seen that the robust stabilization of Cable 1 is higher than that of Cables 2 and 3.

\section{B. Cold Dielectric Type HTS Cable}

Three types of cold dielectric type HTS cable with different optimal parameters are shown in Table IV.

TABLE IV. THERE TyPES OF COLD DiELECTRIC TyPE HTS CABLE CONDUCTORS

\begin{tabular}{|c|c|c|c|c|c|c|c|}
\hline \multicolumn{2}{|c|}{ Layer Index } & NO.1 & NO.2 & NO.3 & NO.4 & NO.5 & NO.6 \\
\hline \multirow{4}{*}{ Cable1 } & $\alpha$ & +1 & +1 & -1 & -1 & +1 & +1 \\
& $\beta$ & $21.2^{\circ}$ & $8.0^{\circ}$ & $8.08^{\circ}$ & $30.0^{\circ}$ & $17.7^{\circ}$ & $8.0^{\circ}$ \\
& $R / m m$ & 9.00 & 9.57 & 10.04 & 10.68 & 17.80 & 18.67 \\
\hline \multirow{4}{*}{ Cable2 } & $\alpha$ & -1 & -1 & +1 & +1 & -1 & +1 \\
& $\beta$ & $20.6^{\circ}$ & $8.3^{\circ}$ & $10.9^{\circ}$ & $3.07^{\circ}$ & $26.0^{\circ}$ & $20.5^{\circ}$ \\
& $R / m m$ & 9.73 & 10.11 & 10.49 & 10.85 & 17.18 & 18.78 \\
\hline \multirow{3}{*}{ Cable3 } & $\alpha$ & -1 & -1 & +1 & +1 & -1 & -1 \\
& $\beta$ & $19.6^{\circ}$ & $8.0^{\circ}$ & $12.0^{\circ}$ & $23.7^{\circ}$ & $23.3^{\circ}$ & $8.3^{\circ}$ \\
& $R / m m$ & 9.11 & 9.73 & 10.47 & 10.86 & 17.22 & 18.87 \\
\hline
\end{tabular}

The perturbations are performed using the same method as before. Fig.11 illustrates the relationship of $E_{\max }$ and winding angle perturbation and radius perturbation respectively.

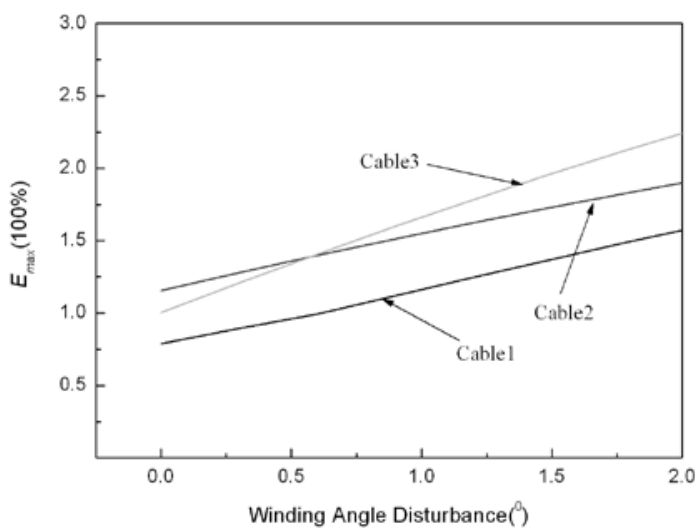

(a) Perturbation of the winding angle

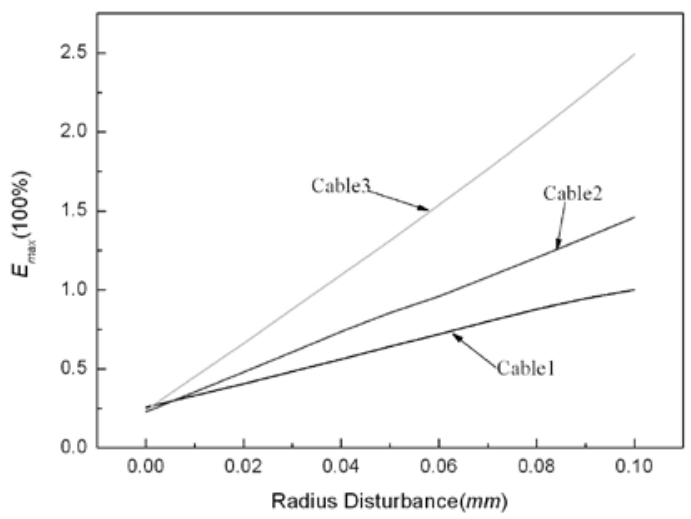

(b) Perturbation of the radius

Figure 11. Influence of parameter perturbation on current distribution in the cold dielectric type HTS cable.

Fig.11 also reveals that $E_{\max }$ of Cable 1 is evidently less than that of Cables 2 and 3. The robust stabilization of Cable 1 is higher than that of Cables 2 and 3.

\section{COMPARISON BETWEEN PSO AND GA}

The following conditions are assumed in the performance comparison between PSO and GA. In one evolution operating, the maximum of generation is set as 5000, and a total of 50 evolutions are conducted for populations of 10, 20 and 40 particles respectively. In this paper, considering the constraints handling, the fitness function described in [12] is applied. Here, $F_{\text {norm }}$ is defined as the average of the best fitness function value of 50 evolutions in the same generation. Only take the 4-layer warm dielectric type HTS power cable for an example.

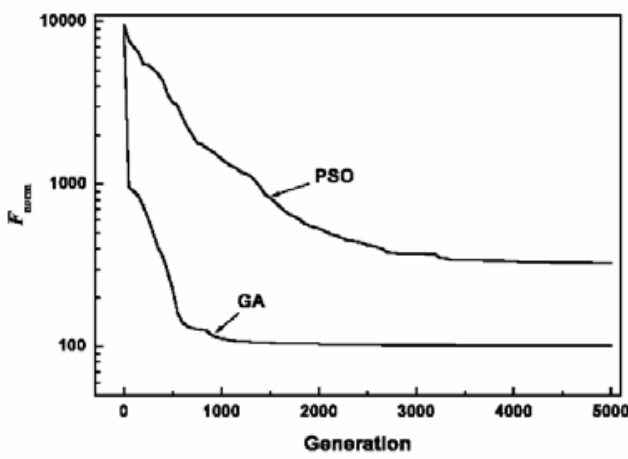

(a) 10 particles 


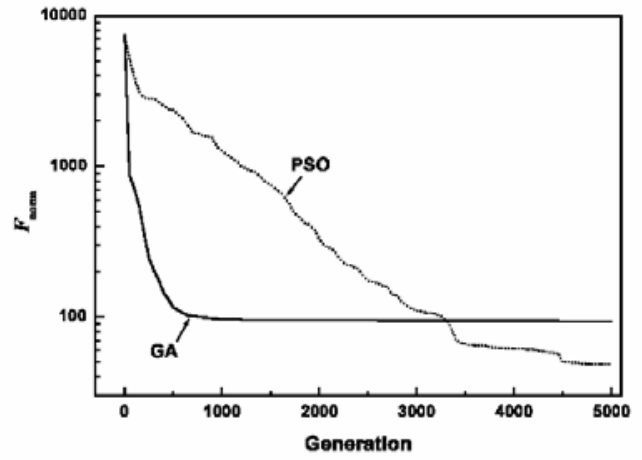

(b) 20 particles

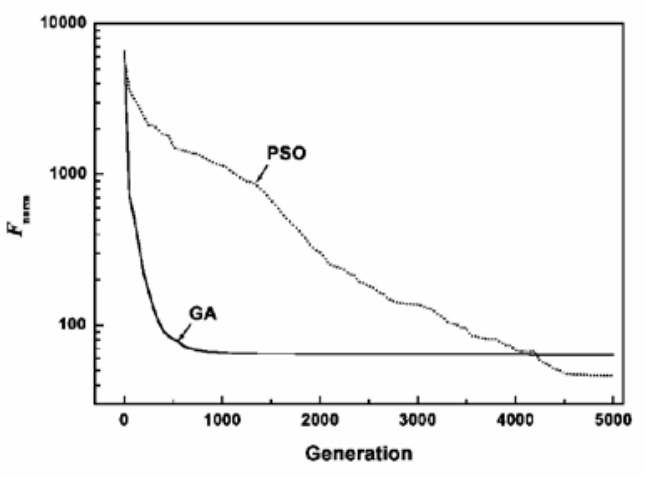

(c) 40 particles

Figure 12. Performance of PSO and GA with different populations.

With reference to the results from Fig.12, GA performs better than PSO when the population is 10 . As the population size reaches 20 and 40, GA also performs better at early generations, but is outpaced by PSO at higher generations. In general, PSO provides a higher performance than that of GA.

The elapsed time of PSO and GA under the same conditions as above is shown in Table V. Evidently, PSO is faster than GA.

TABLE V. SUMMARY OF THE ELAPSED TIME

\begin{tabular}{|c|c|c|}
\hline Population Size & PSO & GA \\
\hline 10 & $28 \mathrm{~s}$ & $73 \mathrm{~s}$ \\
\hline 20 & $44 \mathrm{~s}$ & $123 \mathrm{~s}$ \\
\hline 40 & $76 \mathrm{~s}$ & $235 \mathrm{~s}$ \\
\hline
\end{tabular}

\section{CONCLUSION}

In this paper, a new heuristic algorithm known as PSO is introduced to handle the structural parameter optimization of both warm and cold dielectric type HTS cables for homogeneous distributed layer currents. The optimized results demonstrate that the non-uniform distribution of current is improved considerably and the AC losses are reduced. The performance of PSO algorithm is evaluated in comparison with GA algorithm for a number of random optimization tests. It is proved that the PSO algorithm is an attractive alternative for solving the structural parameter optimization problems. In addition, a perturbation analysis is conducted to evaluate the results after optimization. It is shown that the abilities of parameter perturbation for various multilayer conductors are greatly different.

\section{REFERENCES}

[1] T. Takahashi, et al., "Dielectric properties of $500 \mathrm{~m}$ long HTS power cable,” IEEE Trans. Appl. Supercond., Vol.15, No.2, pp. 1767-1770, June 2005.

[2] Do-Woon Kim, et al. "Development of the 22.9-kV Class HTS Power Cable in LG Cable,” IEEE Trans. Appl. Supercond., vol.15, No.2, June 2005, pp.1723-1726.

[3] S. K. Olsen, C. Træholt, A. Kühle, O. Tønnesen, M. Däumling, J. Østergaard, "Loss and inductance investigations in a 4-layer superconducting prototype cable conductor," IEEE Trans. Appl. Supercond., vol. 9, pp. 833-836, June 1999.

[4] S. Mukoyama, K. Miyoshi, H. Tsubouti, T. Yoshida, M. Mimura, H. Ishii, et al. "Uniform current distribution conductor of HTS power cable with variable tape-winding pitches," IEEE Trans. Appl. Supercond., vol. 9, pp. 1269-1272, June 1999.

[5] V. E. Sytnikov, P. I. Dolgosheev, M. G. Soloviev, D. I. Belij, L.Nieto, A. Perez, et al. "The current test results for two models of HTS cables on CASAT project,” IEEE Trans. Appl. Supercond., vol. 13, pp. 19641967, June 2003.

[6] M. Tsuda, A. K. M. Alamgir, Y. Ito, N. Harada, et al., "Homogeneous Current Distribution in a Coaxial Superconductor with and without Return Current Path,” IEEE Trans. Appl. Supercond., vol. 11, No. 1,pp. 2481-2484, 2001

[7] HNoji, KHaji and T Hamada. "Alternating current loss calculation in a high-TC superconducting transmission cable considering the magnetic field distribution,” Supercond. Sci. Technol. 16 (2003), pp.14-18.

[8] Y. B. Lin, L. Z. Lin, S. P. Li, F. Y. Zhang, S. F. Chen, Y. S. Wang, et al. "Development of a 1000 A class bismuth-based HTS model cable," Chinese Journal of Low Temperature Physics, vol. 21, pp. 122-130, April 1999.

[9] R. Eberhart, J. Kennedy, “A new optimizer using particle swarm theory," 6th International Symposium on Micro Machine and Human Science. Japan, pp. 39-43, 1995.

[10] Y. Shi, R. Eberhart, “A modified particle swarm optimizer,” IEEE International Conference on Evolutionary Computation. USA, pp. 69-73, 1998.

[11] N.Magnusson. "Semi-empirical model of the losses in HTS tapes carrying AC currents in AC magnetic fields applied parallel to the tape face”. Physica C, 2001, vol.349, 2001, pp. 225-234.

[12] Kalyanmoy Deb. "An efficient constraint handling method for genetic algorithm”. Computer methods in applied mechanics and engineering , vol. 186, 2000, pp. 311-338. 